\title{
Decorin levels in early- and late-onset preeclampsia
}

\author{
Gülten Özgen®i), Gültekin Adanaş Aydın®(1) \\ Bursa Yüksek Ihtisas Training and Research Hospital Department of Gynecology and Obstetrics, Bursa, Turkey
}

\begin{abstract}
Objectives: Preeclampsia (PE) is a pregnancy complication caused by typically limited proliferation, apoptosis, migration, and invasion of extra-trophoblast cells. Decorin (DCN) is a decidua-derived transforming growth factor (TGF)-binding proteoglycan which exerts multiple physiological functions such as collagen fibrillogenesis, myogenesis, angiostasis, and restraining placental invasiveness by adversely regulate proliferation, migration, and invasiveness of human extravillous trophoblast cells. Preeclampsia is mainly classified as early-and late-onset PE according to the timing of the disease onset. In the present study, we aimed to investigate the DCN levels in early-onset $P E(E O P E,<34$ weeks) and late-onset severe $P E$ (LOPE, $\geq 34$ weeks) and uncomplicated pregnancies.

Material and methods: In this case-control study, serum samples were obtained from 21 pregnant women with EOPE and 29 pregnant women with LOPE, as well as from 38 healthy controls $(n=12$ early-onset controls and $n=26$ late-onset controls) with uncomplicated pregnancies.

Results: The mean DCN level was statistically significantly higher in the early-onset PE controls than late-onset PE controls $(p=0.040)$. Although the mean DCN level was higher in the early-onset PE controls than EOPE and LOPE groups, it did not reach statistical significance $(p=0.119$ and $p=0.117$, respectively).

Conclusions: Although DCN has been thought to play a role in the pathophysiology of $\mathrm{PE}$, our study results show that $\mathrm{DCN}$ is not a useful predictive marker of EOPE and LOPE. Further large-scale studies are needed to draw a definitive conclusion. Key words: early-onset; late-onset; preeclampsia; decorin
\end{abstract}

Ginekologia Polska 2020; 91, 5: 262-268

\section{INTRODUCTION}

Hypertensive disorders in pregnancy are a major health problem worldwide and preeclampsia (PE) is the most common complication [1]. Preeclampsia accounts for 3 to $5 \%$ of all pregnancies and is one of the leading causes of maternal, fetal, and neonatal mortality and morbidity [2]. It is mainly classified as early-onset ( $<34$ weeks) and late-onset ( $\geq 34$ weeks) [3]. Although initial symptoms are similar in both conditions, they have unique biomarkers, genetic risk factors, prognosis, and clinical characteristics [4].

Decorin (DCN), an extracellular matrix protein, is a small leucine-rich proteoglycan expressed in connective tissue. It contains a protein core and a single chondroitin/dermatan sulfateglycosaminoglycan chain bound at the $\mathrm{N}$-terminal extension. Previous studies have shown that DCN plays a role in the cell proliferation and formation of collagen fibers and modulates certain cell functions (i.e., proliferation, dissemination, migration, and differentiation) acting as a critical modulator of inflammation. In addition,
DCN is a molecule which is highly expressed in reproductive tissues [5-7].

Decorin binds to the transforming growth factor-beta (TGF- $\beta$ ) and activates signaling pathways. The TGF- $\beta$ binds to its own receptor and induces phosphorylation of the Smad family, which is one of the transcription factors, thereby, modulating the transcription of collagen, matrix metalloproteinases (MMPs), and metalloproteinase tissue inhibitors [8]. Irrespective of these mechanisms, DCN stimulates phosphorylation of vascular endothelial growth factor (VEGF) and insulin-like growth (IGF) receptor expressed by extra-villous trophoblasts [9].

In the literature, alterations in the DCN levels have been shown to be associated with PE. In a study, Gogiel et al. [10] reported increased DCN levels of the umbilical cord vein wall in patients with PE. Similarly, Siddiqui et al. [11] found that increased DCN levels were predictors of PE even before the onset of clinical symptoms. The link between DCN and PE can be attributed to the impaired proliferation and migra- 
tion of trophoblasts and endothelial dysfunction, which are thought to be responsible for adverse pregnancy outcomes (APOs). On the other hand, there is a limited number of studies showing the relationship between DCN and APOs with controversial results.

Based on the pathophysiological mechanisms of PE, we hypothesized that DCN would be useful in the diagnosis of $P E$, particularly in early-onset $P E$. In the present study, we, therefore, aimed to investigate the DCN levels in early-onset PE (EOPE) and late-onset PE (LOPE) and uncomplicated pregnancies.

\section{MATERIAL AND METHODS}

This prospective, case-control study was carried out at Bursa Yüksek Ihtisas Training and Research Hospital, Obstetrics and Gynecology outpatient clinics between January 2019 and March 2019. A total of 88 participants aged between 18 and 35 years ( $n=50 P E$ and $n=38$ healthy controls) were included in the study. The patient group was classified as EOPE $(n=21)$ and LOPE $(n=29)$. The control group consisted of healthy women with singleton pregnancy with similar gestational weeks who were under follow-up in our outpatient clinics with uncomplicated pregnancies. Patients with chronic hypertension, thyroid dysfunction, renal or cardiovascular disease, and multiple pregnancy were excluded from the study. Of the control group, 12 were in the $<34^{\text {th }}$ week of pregnancy (early-onset PE controls) and 26 were in the $\geq 34^{\text {th }}$ week of pregnancy (late-onset PE controls). A written informed consent was obtained from each participant. The study protocol was approved by the institutional Ethics Committee (2011-KAEK-25 2019/02-10). The study was conducted in accordance with the principles of the Declaration of Helsinki.

Data including demographic data of the patients, maternal age, parity/gravida, last menstrual period, gestational age, body weight and height, and systolic and diastolic blood pressure were recorded. In those with unknown last menstrual period, the gestational age was calculated based on the crown-rump length as assessed by ultrasound in the first trimester.

The diagnosis of PE was based on a systolic blood pressure of $\geq 140 \mathrm{mmHg}$ or diastolic blood pressure $\geq 90 \mathrm{mmHg}$, measured twice in 4 to 6 -hour intervals while resting, after the $20^{\text {th }}$ gestational week accompanied by $300 \mathrm{mg} / \mathrm{dL}$ proteinuria in a 24-hour urine sample, or more than +1 proteinuria in spot urine specimens. Early-onset PE was defined as the onset before 34 weeks of pregnancy, while late-onset PE was defined as the onset after 34 weeks of pregnancy. The presence of intrauterine growth retardation (IUGR) defined as an estimated fetal weight below the $10^{\text {th }}$ percentile for the gestational age birth.

All patients were followed during pregnancy. Data including birth data, birth weight, and type of labor were recorded.

\section{Biochemical Analyses}

A 5-mL venous blood sample was drawn from each patient during their ward stay and from each healthy control during outpatient visit. The samples were centrifuged at $3,500 \mathrm{rpm}$ for $10 \mathrm{~min}$ and kept at $-80^{\circ}$ until analysis. Serum DCN levels were analyzed using the enzyme-linked immunosorbent (ELISA) method.

Complete blood count and biochemical parameters were analyzed. Complete blood count was analyzed using the Roche SYSMEX analyzer (Roche Diagnostics, Basel, Switzerland). In addition, alanine aminotransferase (ALT), aspartate aminotransferase (AST), lactate dehydrogenase (LDH), total bilirubin, hemoglobin, creatinine, uric acid, and urinalysis were examined using the Synchron LX20 system (Beckman Coulter Diagnostics, CA, USA).

\section{Statistical Analysis}

Statistical analysis was performed using the SPSS version 23.0 software (IBM Corp., Armonk, NY, USA). Descriptive data were expressed in mean \pm standard deviation (SD), quartile $\left(25^{\text {th }}, 50^{\text {th }}\right.$, and $\left.75^{\text {th }}\right)$, and number and frequency. The Kolmogorov-Smirnov test was used to test normal distribution of continuous variables. The Kruskal-Wallis test was used to analyze significant differences between non-normally distributed variables. The post-hoc Dunn test was performed to identify groups with significant differences. The Fisher-Freeman-Halton exact test was used to examine distribution of categorical variables. The Spearman's correlation analysis was carried out to examine the relationship between DCN levels and other variables. A $p$ value of $<0.05$ was considered statistically significant.

\section{RESULTS}

A total of 88 participants including 50 patients with PE and 38 healthy controls were included in this study. Of the patients, 21 had EOPE and 29 had LOPE. Of the healthy controls, 12 were early-onset $P E$ controls and 26 were late-onset PE controls. Demographic and clinical characteristics and biochemical analyses are shown in Table 1.

Although the mean body weight $(p=0.001)$, body mass index $(p=0.006)$, systolic $(p=0.001)$ and diastolic blood pressure $(p=0.001), \operatorname{ALT}(p=0.001)$, hemoglobin $(p=0.016)$, and creatinine $(p=0.001)$ levels did not significantly differ between the either control group, these levels were significantly lower in the control groups than EOPE and LOPE groups. The mean AST level was similar between the control groups and in the LOPE group, but was significantly lower than the EOPE group $(p=0.001)$. On the other hand, there was no significant difference in the platelet counts between the control groups; however, the mean platelet count was significantly higher than the EOPE and LOPE groups. In addition, the mean platelet count was significantly lower in the EOPE group than the LOPE group $(p=0.001)$ 


\begin{tabular}{|c|c|c|c|c|c|c|c|}
\hline & & & EOPE & LOPE & Late-onset PE controls & Early-onset PE controls & $\mathbf{p}$ \\
\hline \multirow{6}{*}{ Age, [year] } & \multicolumn{2}{|l|}{$\mathrm{N}$} & 21 & 29 & 26 & 12 & \multirow{6}{*}{0.082} \\
\hline & \multicolumn{2}{|l|}{ Mean } & 29.76 & 30.72 & 26.62 & 26.42 & \\
\hline & \multicolumn{2}{|l|}{ SD } & 7.293 & 6.403 & 6.682 & 6.735 & \\
\hline & \multirow{3}{*}{ Percentiles } & $25^{\text {th }}$ & 23.50 & 25.50 & 21.75 & 22.00 & \\
\hline & & Median & 29.00 & 32.00 & 25.00 & 23.50 & \\
\hline & & $75^{\text {th }}$ & 37.00 & 36.00 & 31.00 & 32.00 & \\
\hline \multirow{6}{*}{ Weight, [kg] } & \multicolumn{2}{|l|}{$\mathrm{N}$} & 21 & 29 & 26 & 12 & \multirow{6}{*}{0.001} \\
\hline & \multicolumn{2}{|l|}{ Mean } & & $90.48^{a}$ & $76.73^{b}$ & $70.58^{b}$ & \\
\hline & \multicolumn{2}{|l|}{ SD } & & 20.373 & 10.452 & 11.889 & \\
\hline & \multirow{3}{*}{ Percentiles } & $25^{\text {th }}$ & 76.00 & 74.00 & 69.50 & 61.50 & \\
\hline & & Median & 80.00 & 90.00 & 79.00 & 68.00 & \\
\hline & & $75^{\text {th }}$ & 87.50 & 101.00 & 85.00 & 79.50 & \\
\hline \multirow{6}{*}{ Height, [cm] } & \multicolumn{2}{|l|}{$\mathrm{N}$} & 21 & 29 & 26 & 12 & \multirow{6}{*}{0.302} \\
\hline & \multicolumn{2}{|l|}{ Mean } & & 163.41 & 162.88 & 159.25 & \\
\hline & \multicolumn{2}{|l|}{ SD } & & 5.172 & 6.755 & 6.930 & \\
\hline & \multirow{3}{*}{ Percentiles } & $25^{\text {th }}$ & 159.00 & 160.00 & 159.50 & 154.00 & \\
\hline & & Median & 162.00 & 165.00 & 164.00 & 160.00 & \\
\hline & & $75^{\text {th }}$ & 166.00 & 166.50 & 167.25 & 165.00 & \\
\hline \multirow{6}{*}{ BMI, $\left[\mathrm{kg} / \mathrm{m}^{2}\right]$} & \multicolumn{2}{|l|}{$\mathrm{N}$} & 21 & 29 & 26 & 12 & \\
\hline & \multicolumn{2}{|l|}{ Mean } & $31.8625^{a}$ & $33.9284^{a}$ & $28.9815^{b}$ & $27.8364^{b}$ & \\
\hline & SD & & 5.55135 & 7.76778 & 4.10810 & 4.43420 & $0 \cap 06$ \\
\hline & & $25^{\text {th }}$ & 29.1279 & 28.3595 & 26.7589 & 24.2936 & 0.000 \\
\hline & Percentiles & Median & 31.6337 & 34.8944 & 28.3356 & 26.1656 & \\
\hline & & $75^{\text {th }}$ & 34.4410 & 37.5954 & 32.0019 & 32.3027 & \\
\hline & $\mathrm{N}$ & & 21 & 29 & 26 & 12 & \\
\hline & Mean & & $162.86^{a}$ & $157.59^{a}$ & $110.00^{b}$ & $110.00^{b}$ & \\
\hline & SD & & 18.205 & 19.208 & 10.583 & 8.528 & \\
\hline SDR, minting & & $25^{\text {th }}$ & 150.00 & 150.00 & 100.00 & 100.00 & 0.001 \\
\hline & Percentiles & Median & 160.00 & 150.00 & 110.00 & 110.00 & \\
\hline & & $75^{\text {th }}$ & 175.00 & 165.00 & 120.00 & 120.00 & \\
\hline & $\mathrm{N}$ & & 21 & 29 & 26 & 12 & \\
\hline & Mean & & $104.76^{a}$ & $98.97^{b}$ & $65.77^{c}$ & $66.67^{c}$ & \\
\hline $\mathrm{DRP} \mathrm{mm} \mathrm{ma}$ & SD & & 8.136 & 10.805 & 7.575 & 8.876 & 0,001 \\
\hline DD, $11101 \mathrm{l}$ & & $25^{\text {th }}$ & 100.00 & 90.00 & 60.00 & 60.00 & 0.001 \\
\hline & Percentiles & Median & 100.00 & 100.00 & 70.00 & 70.00 & \\
\hline & & $75^{\text {th }}$ & 110.00 & 100.00 & 70.00 & 70.00 & \\
\hline & $\mathrm{N}$ & & 21 & 29 & 26 & 12 & \\
\hline & Mean & & 2.43 & 2.72 & 2.23 & 2.33 & \\
\hline Gravids & SD & & 1.720 & 1.771 & 0.863 & 1.073 & 0076 \\
\hline Miaviua & & $25^{\text {th }}$ & 1.00 & 1.00 & 2.00 & 1.00 & 0.010 \\
\hline & Percentiles & Median & 2.00 & 2.00 & 2.00 & 3.00 & \\
\hline & & $75^{\text {th }}$ & 3.00 & 4.00 & 3.00 & 3.00 & \\
\hline & $\mathrm{N}$ & & 21 & 29 & 26 & 11 & \\
\hline & Mean & & 1.10 & 1.10 & 1.15 & 1.36 & \\
\hline Parity & SD & & 1.221 & 1.263 & 0.881 & 1.206 & 0788 \\
\hline ranty & & $25^{\text {th }}$ & 0.00 & 0.00 & 0.75 & 0.00 & 0.100 \\
\hline & Percentiles & Median & 1.00 & 1.00 & 1.00 & 2.00 & \\
\hline & & $75^{\text {th }}$ & 2.00 & 2.00 & 2.00 & 2.00 & \\
\hline
\end{tabular}




\begin{tabular}{|c|c|c|c|c|c|c|c|}
\hline & & & EOPE & LOPE & Late-onset PE controls & Early-onset PE controls & p \\
\hline \multirow{6}{*}{ Abortus } & \multicolumn{2}{|l|}{ N } & 21 & 29 & 26 & 12 & \multirow{6}{*}{0.057} \\
\hline & \multicolumn{2}{|l|}{ Mean } & 0.33 & 0.62 & 0.08 & 0 & \\
\hline & \multicolumn{2}{|l|}{ SD } & 0.796 & 1.115 & .272 & 0 & \\
\hline & \multirow{3}{*}{ Percentiles } & $25^{\text {th }}$ & 0 & 0 & 0 & 0 & \\
\hline & & Median & 0 & 0 & 0 & 0 & \\
\hline & & $75^{\text {th }}$ & 0 & 1.00 & 0 & 0 & \\
\hline \multirow{6}{*}{ Live birth } & \multicolumn{2}{|l|}{$\mathrm{N}$} & 21 & 29 & 26 & 12 & \multirow{6}{*}{0.635} \\
\hline & \multicolumn{2}{|l|}{ Mean } & 1.10 & 1.10 & 1.15 & 1.50 & \\
\hline & \multicolumn{2}{|l|}{ SD } & 1.221 & 1.263 & 0.881 & 1.243 & \\
\hline & \multirow{3}{*}{ Percentiles } & $25^{\text {th }}$ & 0 & 0 & 0.75 & 0 & \\
\hline & & Median & 1.00 & 1.00 & 1.00 & 2.00 & \\
\hline & & $75^{\text {th }}$ & 2.00 & 2.00 & 2.00 & 2.75 & \\
\hline \multirow{6}{*}{ PLT, [103/mL] } & \multicolumn{2}{|l|}{$\mathrm{N}$} & 21 & 29 & 26 & 12 & \multirow{6}{*}{0.001} \\
\hline & \multicolumn{2}{|l|}{ Mean } & $109.52^{\mathrm{a}}$ & $146.93^{b}$ & $264.73^{c}$ & $272.00^{c}$ & \\
\hline & \multicolumn{2}{|l|}{ SD } & 42.666 & 63.837 & 62.332 & 38.657 & \\
\hline & \multirow{3}{*}{ Percentiles } & $25^{\text {th }}$ & 83.50 & 98.50 & 197.00 & 248.25 & \\
\hline & & Median & 96.00 & 140.00 & 275.00 & 263.00 & \\
\hline & & $75^{\text {th }}$ & 148.00 & 181.00 & 313.00 & 282.50 & \\
\hline \multirow{6}{*}{ WBC, $\left[10^{3} / \mathrm{mL}\right]$} & \multicolumn{2}{|l|}{$\mathrm{N}$} & 21 & 29 & 25 & 12 & \\
\hline & \multicolumn{2}{|l|}{ Mean } & $15.457^{\mathrm{a}}$ & $14.345^{\mathrm{a}}$ & $12.992^{\mathrm{ab}}$ & $10.950^{\mathrm{b}}$ & \\
\hline & SD & & 5.6085 & 4.0497 & 3.4938 & 1.5193 & ד \\
\hline & & $25^{\text {th }}$ & 10.800 & 11.200 & 10.350 & 9.525 & 0.021 \\
\hline & Percentiles & Median & 17.800 & 13.500 & 12.100 & 11.200 & \\
\hline & & $75^{\text {th }}$ & 19.650 & 17.750 & 16.350 & 11.600 & \\
\hline & $N$ & & 21 & 29 & 26 & 12 & \\
\hline & Mean & & $10.6000^{\mathrm{a}}$ & $10.6586^{a}$ & $11.5769^{b}$ & $11.5250^{\mathrm{b}}$ & \\
\hline Hh [a/du] & SD & & 1.44948 & 1.18609 & 1.18061 & 1.25200 & 0016 \\
\hline $\mathrm{HD},[\mathrm{g} / \mathrm{dL}]$ & & $25^{\text {th }}$ & 9.6000 & 9.8000 & 10.9000 & 10.4250 & 0.016 \\
\hline & Percentiles & Median & 10.9000 & 10.9000 & 11.8000 & 11.3000 & \\
\hline & & $75^{\text {th }}$ & 11.6000 & 11.5500 & 12.5000 & 12.6000 & \\
\hline & $N$ & & 21 & 29 & 26 & 12 & \\
\hline & Mean & & $115.7619^{a}$ & $61.3448^{b}$ & $20.2308^{b}$ & $19.4167^{b}$ & \\
\hline & SD & & 66.05067 & 40.55972 & 8.18441 & 4.01040 & \\
\hline AST, [IU/L] & & $25^{\text {th }}$ & 50.0000 & 27.5000 & 13.5000 & 16.2500 & 0.001 \\
\hline & Percentiles & Median & 121.0000 & 46.0000 & 18.5000 & 19.0000 & \\
\hline & & $75^{\text {th }}$ & 152.0000 & 93.5000 & 25.0000 & 22.0000 & \\
\hline & $N$ & & 21 & 29 & 26 & 12 & \\
\hline & Mean & & $111.3810^{\mathrm{a}}$ & $53.3207^{b}$ & $16.2692^{c}$ & $12.6667^{c}$ & \\
\hline AIT [IIII & SD & & 76.59209 & 48.74625 & 9.15885 & 3.65148 & 1001 \\
\hline$A L I,[U / L]$ & & $25^{\text {th }}$ & 26.0000 & 21.0000 & 9.7500 & 10.2500 & 0.001 \\
\hline & Percentiles & Median & 117.0000 & 35.0000 & 13.5000 & 12.0000 & \\
\hline & & $75^{\text {th }}$ & 154.0000 & 70.0000 & 23.0000 & 15.7500 & \\
\hline & $\mathrm{N}$ & & 21 & 29 & 26 & 12 & \\
\hline & Mean & & $20.8095^{a}$ & $15.3310^{b}$ & $8.6231^{c}$ & $6.4333^{d}$ & \\
\hline Urea $[\mathrm{ma} / \mathrm{d}$ & SD & & 6.87836 & 5.97764 & 2.37627 & 2.45872 & 0001 \\
\hline Urea, [mg/dL] & & $25^{\text {th }}$ & 14.7000 & 10.7000 & 7.1750 & 4.6000 & 0.001 \\
\hline & Percentiles & Median & 21.7000 & 15.0000 & 8.4500 & 6.1000 & \\
\hline & & $75^{\text {th }}$ & 25.7000 & 18.3000 & 10.0500 & 8.0000 & \\
\hline
\end{tabular}


Table 1. Demographic and clinical characteristics and biochemical analyses

\begin{tabular}{|c|c|c|c|c|c|c|c|}
\hline & & & EOPE & LOPE & Late-onset PE controls & Early-onset PE controls & p \\
\hline \multirow{6}{*}{$\begin{array}{l}\text { Creatinine, } \\
{[\mathrm{mg} / \mathrm{dL}]}\end{array}$} & \multicolumn{2}{|l|}{$\mathrm{N}$} & 21 & 29 & 26 & 12 & \multirow{6}{*}{0.001} \\
\hline & \multicolumn{2}{|l|}{ Mean } & $1.0648^{\mathrm{a}}$ & $.9500^{\mathrm{a}}$ & $.6688^{b}$ & $.6558^{\mathrm{b}}$ & \\
\hline & \multicolumn{2}{|l|}{ SD } & .33898 & .31988 & .14605 & .10757 & \\
\hline & \multirow{3}{*}{ Percentiles } & $25^{\text {th }}$ & 0.7450 & 0.7100 & 0.5950 & 0.6025 & \\
\hline & & Median & 1.1000 & 0.8300 & 0.6300 & 0.6750 & \\
\hline & & $75^{\text {th }}$ & 1.2650 & 1.2000 & 0.7000 & 0.7175 & \\
\hline \multirow{6}{*}{ Gestational week } & \multicolumn{2}{|l|}{$\mathrm{N}$} & 21 & 29 & 26 & 12 & \multirow{6}{*}{0.001} \\
\hline & \multicolumn{2}{|l|}{ Mean } & $30.48^{\mathrm{a}}$ & $35.90^{b}$ & $37.12^{c}$ & $37.42^{c}$ & \\
\hline & \multicolumn{2}{|l|}{ SD } & 2.462 & 1.566 & 1.532 & 2.065 & \\
\hline & \multirow{3}{*}{ Percentiles } & $25^{\text {th }}$ & 28.50 & 35.00 & 36.00 & 37.00 & \\
\hline & & Median & 32.00 & 36.00 & 37.00 & 38.00 & \\
\hline & & $75^{\text {th }}$ & 32.00 & 37.00 & 38.25 & 39.00 & \\
\hline \multirow{6}{*}{ Birth weight, [g] } & \multicolumn{2}{|l|}{$\mathrm{N}$} & 20 & 29 & 26 & 12 & \multirow{6}{*}{0.001} \\
\hline & \multicolumn{2}{|l|}{ Mean } & $1239.25^{\mathrm{a}}$ & $2572.45^{b}$ & $3044.23^{c}$ & $3003.33^{c}$ & \\
\hline & \multicolumn{2}{|l|}{ SD } & 475.448 & 722.840 & 581.914 & 622.405 & \\
\hline & \multirow{3}{*}{ Percentiles } & $25^{\text {th }}$ & 847.50 & 1965.00 & 2460.00 & 2912.50 & \\
\hline & & Median & 1215.00 & 2530.00 & 3160.00 & 3150.00 & \\
\hline & & $75^{\text {th }}$ & 1527.50 & 3275.00 & 3490.00 & 3350.00 & \\
\hline \multirow{6}{*}{ Decorin, [pg/mL] } & \multicolumn{2}{|l|}{$\mathrm{N}$} & 21 & 29 & 26 & 12 & \multirow{6}{*}{0.040} \\
\hline & \multicolumn{2}{|l|}{ Mean } & 10.8524 & 11.0276 & 9.9750 & 14.4250 & \\
\hline & \multicolumn{2}{|l|}{ SD } & 4.34714 & 3.86577 & 4.56240 & 5.24632 & \\
\hline & \multirow{3}{*}{ Percentiles } & $25^{\text {th }}$ & 7.4000 & 8.4500 & 8.0000 & 10.8250 & \\
\hline & & Median & 10.2000 & 9.5000 & 10.1000 & 13.8000 & \\
\hline & & 75th & 15.0000 & 13.8000 & 11.8750 & 19.5000 & \\
\hline
\end{tabular}

EOPE — early-onset preeclampsia; LOPE — late-onset preeclampsia; PE — preeclampsia; SD — standard deviation; BMI — body mass index; SBP — systolic blood pressure; DBP — diastolic blood pressure; PLT— platelet; WBC — white blood cell; Hb— hemoglobin; AST — aspartate aminotransferase; ALT— alanine aminotransferase

The mean DCN level was statistically significantly higher in the early-onset PE controls than late-onset PE controls $(p=0.040)$. Although the mean DCN level was higher in the early-onset PE controls than the EOPE and LOPE groups, it did not reach statistical significance $(p=0.119$ and $p=0.117$, respectively). However, based on the $p$ values of these variables, the difference between the groups may be of biological relevance, although not statistically significant.

\section{DISCUSSION}

Preeclampsia is one of the complications of pregnancy and is mainly classified into two types according to the time of occurrence: early-onset PE ( $<34^{\text {th }}$ week of pregnancy) and late-onset PE ( $\geq 34^{\text {th }}$ week of pregnancy). 'Early-onset PE occurs in about $10 \%$ of all preeclamptic cases and has a complex pathophysiology, the main cause being abnormal placentation with maternal predictive factors. It seems, therefore, reasonable to gain a better understanding of the underlying angiogenic imbalance in early- and late-onset PE and to identify and treat candidate patients at the end of the first trimester, as the incidence of maternal vascular malperfusion and placental vascular lesions are higher in early-onset PE [12].

Failed trophoblast invasion has been proposed the main pathogenetic mechanism in PE. Previous studies have demonstrated that PE is a two-stage disorder: abnormal placentation with reduced placental perfusion in the first stage and maternal systemic pathophysiological changes in the second stage. However, the exact underlying mechanism of the lack of invasion of extravillous trophoblasts in PE remains to be elucidated [13].

Implantation and placentation are essential components of pregnancy which thoroughly rely upon fundamental biological processes invasive trophoblasts, growth factors, growth factor binding proteins, proinflammatory cytokines, proteoglycans, and including highly MMPs. The regulation of MMP activity is the mainstay of these critical processes. Dysregulation of these delicate processes may result in a broad range of pregnancy abnormalities such as PE, IUGR, preterm labor, and miscarriage [14]. 
In the early period of pregnancy, the fetoplacental development is mediated by a complex cascade system containing growth factors, cytokines, and transcription factors [15]. Decorin is a product of both fetal mesenchymal cells within the placenta and decidual cells in the endometrium. Currently, the role of DCN in stem cell regulation and in the underlying pathogenesis of PE and IUGR has not been fully elucidated. During a recent study, Siddiqui et al. [11] investigated the relation of DCN overexpression in the chorionic villi and/or basal decidua with PE. They reported that basal decidual cell-induced DCN overexpression was related to hypoinvasive phenotype with poor endovascular trophoblast cell differentiation in PE. In addition, the authors found no significant change in DCN levels depending on gestational age during the second trimester in PE patients, although there was an inverse association between the plasma DCN levels and body mass index or body weight. Based on these findings, the authors concluded that increased plasma DCN level might be a predictor of PE before the onset of clinical signs. In another study, Siddiqui et al. [11] found that DCN messenger ribonucleic acid (mRNA) expression at the cellular level showed significantly increased expression in basal plate decidual cells within the placentas from PE ( 23 to 40 weeks of gestation) patients than controls at all gestational age. Similarly, Nandi et al. [16] found a significant difference in the DCN staining of placental tissues between the PE and control groups. However, at the tissue level, DCN mRNA expression in chorionic villi was similar. In another study, Nandi et al. [17] reported that elevated DCN levels in the maternal blood could be a predictive biomarker for PE.

For a healthy pregnancy, the maternal blood vessel remodeling is driven by the extravillous cytotrophoblasts rather than maternal endothelium. Reduced interstitial invasion and endovascular cytotrophoblasts are associated with IUGR. In their study, Weber et al. [18] described a variety of trophoblast stem cell and pluripotency marker staining patterns based on gestational age and placenta-associated pregnancy complications. The authors concluded that PE, IUGR, and combined PE + IUGR are separate entities based on the differential expression patterns within the placentas complicated with placenta-associated pregnancy complications. We believe that reduced DCN may lead to uncontrolled proliferation and inadequate differentiation of cytotrophoblasts, thereby, resulting in impaired ion-nutrition exchange and decreased hormonal synthesis. More importantly, differentiation of cytotrophoblasts is the cornerstone of healthy placental development in human [15].

In a study, Tan et al. [19] found that abnormal differentiation of trophoblast stem cells was likely to be associated with IUGR. Since certain types of IUGR and PE share a common placental pathology, the authors concluded that overexpres- sion of DCN in the placenta/decidua led to poor trophoblast differentiation in an IUGR subgroup.

Caglar et al. [20] compared DCN levels between pregnancies complicated by idiopathic IUGR and uncomplicated pregnancies and examined the possible relationship between DCN levels and clinical parameters. They found significantly higher maternal serum DCN levels in complicated pregnancies by IUGR and an about 8-times higher risk of high maternal serum DCN levels in complicated pregnancies.

In a study, Murthi et al. [21] collected first trimester tissues via chorionic villus sampling and investigated the temporal relationship between subsequent development of small for gestational age (SGA) and altered DCN expression. The DCN mRNA were determined via using real-time polymerase chain reaction (PCR) and DCN proteins via immunoblotting. The authors showed that DCN mRNA and protein significantly decreased in the placentas from the first-trimester SGA-pregnancies. The aforementioned study is to the first to report a temporal relationship between subsequent development of SGA and altered placental DCN expression in the literature. Similarly, in a previous study of the same researchers, the DCN expression significantly reduced in IUGR compared to gestation-matched controls [22].

The mean DCN level was statistically significantly higher in the early-onset PE controls than late-onset PE controls $(p=0.040)$. Although the mean DCN level was higher in the early-onset PE controls than EOPE and LOPE groups, it did not reach statistical significance $(p=0.119$ and $p=0.117$, respectively). However, based on the $p$ values of these variables, we suggest that the difference between the groups may be of biological relevance, although not statistically significant.

Nonetheless, there are some limitations to this study. First, due to the prospective design of the study and termination of the data cut-off date, the number of patients in the control group cannot be increased. Second, we were unable to perform immunohistochemical staining for DCN expression of placental tissues. Despite the lack of any statistically significant difference in the maternal serum DCN samples between the early-onset and late-onset PE groups, no data are available whether there is a significant difference in the DCN level of placental tissues due to the lack of immunohistochemical stating.

In conclusion, although DCN has been thought to be involved in the pathophysiology of $\mathrm{PE}$, our study results show that DCN is not a useful predictive marker of EOPE and LOPE. However, these results might have been yielded due to small sample size of our study. Therefore, further large-scale studies are needed to draw a definitive conclusion. Furthermore, it would be more helpful to gain an insight into the role of DCN in the pathophysiology of PE by measuring the DCN mRNA expression in the basal plate decidual cells within the placenta with immunohistochemical staining. 


\section{Conflict of interest}

The authors declare no conflict of interest. The authors are solely responsible for the content and writing of the paper.

\section{Financial disclosure}

The authors receive no financial support for the study conduct.

\section{Ethical disclosure}

A written informed consent was obtained from each participant. The study protocol was approved by the Ethics Committee of Bursa Yüksekihtisas Training and Research Hospital. The study was conducted in accordance with the principles of the Declaration of Helsinki.

\section{Confidentiality of data}

All authors of this manuscript declare that they have followed the protocols of publication of patient's data. All caregivers of the participants were informed in detail about the research and signed patient informed consent.

\section{REFERENCES}

1. Wallis AB, Saftlas AF, Hsia J, et al. Secular trends in the rates of preeclampsia, eclampsia, and gestational hypertension, United States, 1987-2004. Am J Hypertens. 2008; 21(5): 521-526, doi: 10.1038/ajh.2008.20, indexed in Pubmed: 18437143.

2. Saleem S, McClure EM, Goudar SS, et al. Global Network Maternal Newborn Health Registry Study Investigators. A prospective study of maternal, fetal and neonatal deaths in low- and middle-income countries. Bull World Health Organ. 2014; 92(8): 605-612, doi: 10.2471/BLT.13.127464, indexed in Pubmed: 25177075.

3. Lisonkova S, Sabr Y, Mayer C, et al. Maternal morbidity associated with early-onset and late-onset preeclampsia. Obstet Gynecol.2014;124(4):771-781, doi: 10.1097/AOG.0000000000000472, indexed in Pubmed: 25198279.

4. Stepan $\mathrm{H}$, Unversucht $\mathrm{A}$, Wessel $\mathrm{N}$, et al. Predictive value of maternal angiogenic factors in second trimester pregnancies with abnormal uterine perfusion. Hypertension. 2007; 49(4): 818-824, doi: 10.1161/01. HYP.0000258404.21552.a3, indexed in Pubmed: 17261644.

5. Krusius T, Ruoslahti E. Primary structure of an extracellular matrix proteoglycan core protein deduced from cloned cDNA. Proc Natl Acad Sci U S A. 1986; 83(20): 7683-7687, doi: 10.1073/pnas.83.20.7683, indexed in Pubmed: 3484330.

6. Schaefer L, lozzo RV. Biological functions of the small leucine-rich proteoglycans: from genetics to signal transduction. J Biol Chem. 2008; 283(31): 21305-21309, doi: 10.1074/jbc.R800020200, indexed in Pubmed: 18463092.

7. Reed CC, lozzo RV. The role of decorin in collagen fibrillogenesis and skin homeostasis. Glycoconj J. 2002; 19(4-5): 249-255, doi: 10.1023/A:1025383913444, indexed in Pubmed: 12975602.
8. Kinsella MG, Bressler SL, Wight TN. The regulated synthesis of versican, decorin, and biglycan: extracellular matrix proteoglycans that influence cellular phenotype. Crit Rev Eukaryot Gene Expr. 2004; 14(3): 203-234 doi: 10.1615/critreveukaryotgeneexpr.v14.i3.40, indexed in Pubmed: 15248816.

9. lacob D, Cai J, Tsonis M, et al. Decorin-mediated inhibition of proliferation and migration of the human trophoblast via different tyrosine kinase receptors. Endocrinology. 2008; 149(12):6187-6197, doi: 10.1210/en.20080780, indexed in Pubmed: 18703624.

10. Gogiel T, Galewska Z, Romanowicz L, et al. Pre-eclampsia-associated alterations in decorin, biglycan and versican of the umbilical cord vein wall. Eur J Obstet Gynecol Reprod Biol. 2007; 134(1):51-56, doi: 10.1016/j. ejogrb.2006.10.003, indexed in Pubmed: 17097211.

11. Siddiqui MF, Nandi P, Girish GV, et al. Decorin over-expression by decidual cells in preeclampsia: a potential blood biomarker. Am J Obstet Gynecol. 2016; 215(3): 361.e1-361.e15, doi: 10.1016/j.ajog.2016.03.020, indexed in Pubmed: 27001218.

12. van der Merwe JL, Hall DR, Wright C, et al. Are early and late preeclampsia distinct subclasses of the disease--what does the placenta reveal? Hypertens Pregnancy. 2010; 29(4):457-467, doi: 10.3109/10641950903572282, indexed in Pubmed: 20701467.

13. Xu G, Guimond MJ, Chakraborty C, et al. Control of proliferation, migration, and invasiveness of human extravillous trophoblast by decorin, a decidual product. Biol Reprod. 2002; 67(2): 681-689, doi: 10.1095/biolreprod67.2.681, indexed in Pubmed: 12135914.

14. Zhu JY, Pang ZJ, Yu YH. Regulation of trophoblast invasion: the role of matrix metalloproteinases. Rev Obstet Gynecol. 2012; 5(3-4): e137-e143, indexed in Pubmed: 23483768.

15. Huppertz B, Frank HG, Kingdom JC, et al. Villous cytotrophoblast regulation of the syncytial apoptotic cascade in the human placenta. Histochem Cell Biol. 1998; 110(5): 495-508, doi: 10.1007/s004180050311, indexed in Pubmed: 9826129.

16. Nandi P, Siddiqui MF, Lala PK. Restraint of Trophoblast Invasion of the Uterus by Decorin: Role in Pre-eclampsia. Am J Reprod Immunol. 2016; 75(3): 351-360, doi: 10.1111/aji.12449, indexed in Pubmed: 26554635.

17. Nandi $\mathrm{P}$, Lim H, Torres-Garcia EJ, et al. Human trophoblast stem cell self-renewal and differentiation: Role of decorin. Sci Rep. 2018; 8(1): 8977, doi: 10.1038/s41598-018-27119-4, indexed in Pubmed: 29895842.

18. Weber M, Göhner C, San Martin S, et al. Unique trophoblast stem celland pluripotency marker staining patterns depending on gestational age and placenta-associated pregnancy complications. Cell Adh Migr. 2016; 10(1-2): 56-65, doi: 10.1080/19336918.2016.1142035, indexed in Pubmed: 26914354.

19. Tan KH, Tan SS, Ng MJ, et al. Extracellular vesicles yield predictive pre-eclampsia biomarkers. J Extracell Vesicles. 2017; 6(1): 1408390, doi: 10.1080/20013078.2017.1408390, indexed in Pubmed: 29296254.

20. Cağlar M, Yavuzcan A, Göksu M, et al. Decorin: a possible marker for fetal growth restriction. Gynecol Endocrinol. 2014; 30(2): 141-144, doi: 10.3109/09513590.2013.860125, indexed in Pubmed: 24256371.

21. Murthi P, van Zanten DE, Eijsink JJH, et al. Decorin expression is decreased in first trimester placental tissue from pregnancies with small for gestation age infants at birth. Placenta. 2016; 45: 58-62, doi: 10.1016/j. placenta.2016.07.008, indexed in Pubmed: 27577711.

22. Swan BC, Murthi P, Rajaraman G, et al. Decorin expression is decreased in human idiopathic fetal growth restriction. Reprod Fertil Dev. 2010; 22(6): 949-955, doi: 10.1071/RD09240, indexed in Pubmed: 20591329. 